\title{
On the Connectivity of Wireless Network Systems and an Application in Teacher-Student Interactive Platforms
}

\author{
Xun Ge \\ School of Mathematical Sciences, Soochow University, Suzhou 215006, China \\ Correspondence should be addressed to Xun Ge; zhugexun@163.com
}

Received 3 April 2014; Revised 5 June 2014; Accepted 6 June 2014; Published 18 June 2014

Academic Editor: Chong Lin

Copyright (C) 2014 Xun Ge. This is an open access article distributed under the Creative Commons Attribution License, which permits unrestricted use, distribution, and reproduction in any medium, provided the original work is properly cited.

\begin{abstract}
A wireless network system is a pair $(U ; \mathscr{B})$, where $\mathscr{B}$ is a family of some base stations and $U$ is a set of their users. To investigate the connectivity of wireless network systems, this paper takes covering approximation spaces as mathematical models of wireless network systems. With the help of covering approximation operators, this paper characterizes the connectivity of covering approximation spaces by their definable subsets. Furthermore, it is obtained that a wireless network system is connected if and only if the relevant covering approximation space has no nonempty definable proper subset. As an application of this result, the connectivity of a teacher-student interactive platform is discussed, which is established in the School of Mathematical Sciences of Soochow University. This application further demonstrates the usefulness of rough set theory in pedagogy and makes it possible to research education by logical methods and mathematical methods.
\end{abstract}

\section{Introduction}

In this paper, we discuss the wireless network system $(U ; \mathscr{B})$ (see Definition 1), where $U$ denotes the set of all users and $\mathscr{B}$ denotes the family of all stations (or servers). For the wireless network system $(U ; \mathscr{B})$, how can we guarantee that any pair of users $u, v$ in $U$ can receive and send information from and to each other? It is an interesting question. Note that a pair of users $u, v$ in $U$ can receive and send information from and to each other if there is a base station $B \in \mathscr{B}$ such that not only $u$ and $B$ but also $v$ and $B$ are connected. Thus, the connectivity for wireless network systems (see Definition 2) is worthy to be considered. How can we investigate the connectivity of a wireless network system $(U ; \mathscr{B})$ ? It is necessary to analyze data collected from $(U ; \mathscr{B})$. Just as stated by Zhu and Wang in [1], "Across a wide variety of fields, data are being collected and accumulated at a dramatic pace, especially in the age of the Internet. There is much useful information hidden in the accumulated voluminous data, but it is very hard for us to obtain it. Thus, there is an urgent need for a new generation of computational theories and tools to assist humans in extracting knowledge from the rapidly growing volumes of digital data; otherwise, these huge data are useless for us." In order to extract and analyze useful information hidden in voluminous data, many methods in addition to classical logic and classical mathematics have been proposed. Rough set theory, which was proposed by Pawlak in [2], plays an important role in applications of these methods. Their usefulness has been demonstrated by many successful applications in information sciences and computer sciences (see, e.g., [2-12]). In particular, rough set theory can handle some information systems with voluminous data. This makes it possible to analyze and compute voluminous data by computer technology. In the past years, with development of information sciences and computer sciences, applications of rough set theory have been extended from Pawlak approximation spaces to covering approximation spaces (see, e.g., [1, 13-23]). It leads us to investigate the connectivity of wireless network systems by covering approximation spaces.

In this paper, we establish some relations between wireless network systems and covering approximation spaces. By these relations, we take covering approximation spaces as mathematical models of wireless network systems and convert investigations of the connectivity from wireless network systems to covering approximation spaces. With the help of covering approximation operators, we characterize the connectivity of covering approximation spaces by their definable subsets. Furthermore, we obtain that a wireless 
network system is connected if and only if the relevant covering approximation space has no nonempty definable proper subset. As an application of this result, the connectivity of a teacher-student interactive platform is discussed, which is established in the School of Mathematical Sciences of Soochow University. This application further demonstrates the usefulness of rough set theory in pedagogy and makes it possible to research education by logical methods and mathematical methods.

\section{Preliminaries}

At first, we describe a wireless network system and its connectivity as follows.

Definition 1. Let $\mathscr{B}$ be a family of some base stations and let $U$ be a set of their users. Then the pair $(U ; \mathscr{B})$ is called a wireless network system if the following conditions are satisfied.

(1) For each user $u$ in $U$, there is a base station $B$ in $\mathscr{B}$ such that $u$ and $B$ are connected.

(2) For each base station $B$ in $\mathscr{B}$, there is a user $u$ in $U$ such that $u$ and $B$ are connected.

Here, $u$ and $B$ are connected if they can receive and send information from and to each other.

The wireless network system $(U ; \mathscr{B})$ stated as above is different from some existing network systems. For example, Soochow University network consists of a central network station $B$ and some users accesses, which is more complicated in structure. All users, who contact each other by the Soochow University network, must connect users accesses with the central network station $B$. However, the wireless network system $(U ; \mathscr{B})$ can make users contact each other by connecting users accesses with some simple base stations. In addition, the wireless network system $(U ; \mathscr{B})$ can show some advantages on network security. That is, the wireless network system $(U ; \mathscr{B})$ has some $S_{i}$-securities (see, e.g., [14]).

Definition 2. Let $(U ; \mathscr{B})$ be a wireless network system.

(1) For two users $u, v \in U, u$ and $v$ are called to have a contact if there are some users $u_{1}, u_{2}, \ldots, u_{n} \in U$ and some base stations $B_{1}, B_{2}, \ldots, B_{n-1} \in \mathscr{B}$ such that, for each $i=1,2, \ldots, n-1$, not only $u_{i}$ and $B_{i}$ but also $u_{i+1}$ and $B_{i}$ are connected, where $u_{1}=u$ and $u_{n}=v$.

(2) $(U ; \mathscr{B})$ is called connected if $u$ and $v$ have a contact for all users $u, v \in U$.

Definition 3 (see [13]). Let $U$, the universe of discourse, be a finite set and let $\mathscr{C}$ be a family of nonempty subsets of $U$.

(1) $\mathscr{C}$ is called a cover of $U$ if $\bigcup\{K: K \in \mathscr{C}\}=U$.

(2) The pair $(U ; \mathscr{C})$ is called a covering approximation space if $\mathscr{C}$ is a cover of $U$.

The following covering approximation spaces will play an important role in our discussion.
Remark 4. (1) A covering approximation space $(U ; \mathscr{C})$ is a Pawlak approximation space if $\mathscr{C}$ is a partition on $U$; that is, elements of $\mathscr{C}$ are mutually disjoint.

(2) A covering approximation space $(U ; \mathscr{C})$ is a generalized topological space if $\emptyset \in \mathscr{C}$ and $\mathscr{C}$ is closed with respect to the union of elements of $\mathscr{C}[24]$, and $(U ; \mathscr{C})$ is a topological space if $\emptyset \in \mathscr{C}$ and $\mathscr{C}$ is closed with respect to both the union and the finite intersection of elements of $\mathscr{C}$ [25].

Proposition 5. Let $(U ; \mathscr{B})$ be a wireless network system. For each base station $B$ in $\mathscr{B}$, let $K_{B}$ be a set of some users in $U$ such that $u$ is a user in $K_{B}$ if and only if $u$ and $B$ are connected. Put $\mathscr{C}=\left\{K_{B}: B \in \mathscr{B}\right\}$. Then $(U ; \mathscr{C})$ is a covering approximation space.

Proof. It suffices to prove that $\mathscr{C}$ is a cover of $U$. Let $u \in U$; that is, $u$ is a user in $U$. By Definition $1(1)$, there is a base station $B$ in $\mathscr{B}$ such that $u$ and $B$ are connected. So $u$ is a user in $K_{B}$; that is, $u \in K_{B}$. This proves that $\mathscr{C}$ is a cover of $U$.

Definition 6. Let $(U ; \mathscr{B})$ be a wireless network system, and let $(U ; \mathscr{C})$ be a covering approximation space described as in Proposition 5 . Then $(U ; \mathscr{C})$ is called to be induced by $(U ; \mathscr{B})$.

In order to convert investigations of the connectivity from wireless network systems to covering approximation spaces, the following "chain" in covering approximation spaces is introduced, the idea of which comes from topology [25].

Definition 7 . Let $(U ; \mathscr{C})$ be a covering approximation space and let $u, v \in U$.

(1) A subfamily $\left\{K_{1}, K_{2}, \ldots, K_{n}\right\}$ of $\mathscr{C}$ is called a chain between $u$ and $v$ if $u \in K_{1}, v \in K_{n}$, and $K_{i} \cap K_{i+1} \neq \emptyset$ for each $i=1,2, \ldots, n-1$.

(2) $u$ is called to be chain connected to $v$ if there is a chain between $u$ and $v$.

Remark 8 . Let $(U ; \mathscr{C})$ be a covering approximation space. Then the relation for "chain connected" is an equivalent relation; that is, the following hold for all $u, v, w \in U$.

(1) $u$ is chain connected to $u$.

(2) $u$ is chain connected to $v$, which implies that $v$ is chain connected to $u$.

(3) $u$ is chain connected to $v$ and $v$ is chain connected to $w$, which implies that $u$ is chain connected to $w$.

Proof. Obviously, (1) and (2) hold. Let $u$ be chain connected to $v$, and let $v$ be chain connected to $w$. Then there are $K_{1}, K_{2}, \ldots, K_{n} \in \mathscr{C}$ such that $u \in K_{1}, v \in K_{n}$, and $K_{i} \cap K_{i+1} \neq \emptyset$ for each $i=1,2, \ldots, n-1$; and there are $K_{n+1}, K_{n+2}, \ldots, K_{n+m} \in \mathscr{C}$ such that $v \in K_{n+1}, w \in K_{n+m}$, and $K_{n+i} \bigcap K_{n+i+1} \neq \emptyset$ for each $i=1,2, \ldots, m-1$. Consequently, there are $K_{1}, K_{2}, \ldots, K_{n}, K_{n+1}, K_{n+2}, \ldots, K_{n+m} \in \mathscr{C}$ such that $u \in K_{1}, w \in K_{n+m}$, and $K_{i} \bigcap K_{i+1} \neq \emptyset$ for each $i=1,2, \ldots, n+$ $m-1$. This proves that $u$ is chain connected to $w$. So (3) holds. 
We give the connectivity of covering approximation spaces.

Definition 9. Let $(U ; \mathscr{C})$ be a covering approximation space. $(U ; \mathscr{C})$ is called connected if, for each pair $u, v \in U$, there is a chain between $u$ and $v$.

Lemma 10. Let $(U ; \mathscr{B})$ be a wireless network system, and let $(U ; \mathscr{C})$ be a covering approximation space induced by $(U ; \mathscr{B})$. Then the following are equivalent for all $u, v \in U$.

(1) $u$ and $v$ have a contact.

(2) There is a chain between $u$ and $v$.

Proof. (1) $\Rightarrow$ (2): let $u$ and $v$ have a contact. Then there are some users $u_{1}, u_{2}, \ldots, u_{n} \in U$ and some base stations $B_{1}, B_{2}, \ldots, B_{n-1} \in \mathscr{B}$ such that, for each $i=1,2, \ldots, n-1$, not only $u_{i}$ and $B_{i}$ but also $u_{i+1}$ and $B_{i}$ are connected, where $u_{1}=u$ and $u_{n}=v$. Since $(U ; \mathscr{C})$ is induced by $(U ; \mathscr{B})$, for each $i=1,2, \ldots, n-1, K_{B_{i}} \in \mathscr{C}$, put $K_{i}=K_{B_{i}}$. Then $K_{1}, K_{2}, \ldots, K_{n-1} \in \mathscr{C}$, and, for each $i=1,2, \ldots, n-1$, $u_{i}, u_{i+1} \in K_{i}$. It follows that $u=u_{1} \in K_{1}, v=u_{n} \in K_{n-1}$, and, for each $i=1,2, \ldots, n-2, u_{i+1} \in K_{i} \bigcap K_{i+1} \neq \emptyset$. This shows that $K_{1}, K_{2}, \ldots, K_{n-1}$ is a chain between $u$ and $v$.

(2) $\Rightarrow$ (1): let $K_{1}, K_{2}, \ldots, K_{n}$ be a chain between $u$ and $v$, that is, $u \in K_{1}$ and $v \in K_{n}$, and $K_{i} \cap K_{i+1} \neq \emptyset$ for each $i=1,2, \ldots, n-1$. Put $u_{1}=u, u_{n+1}=v$, and, for each $i=1,2, \ldots, n-1$, choose $u_{i+1} \in K_{i} \cap K_{i+1}$. It follows that $u_{i}, u_{i+1} \in K_{i}$ for each $i=1,2, \ldots, n$. Since $(U ; \mathscr{C})$ is induced by $(U ; \mathscr{B})$, there are base stations $B_{1}, B_{2}, \ldots, B_{n} \in \mathscr{B}$ such that $K_{i}=K_{B_{i}}$ for each $i=1,2, \ldots, n$. Thus, for each $i=1,2, \ldots, n$, $u_{i}, u_{i+1} \in K_{B_{i}}$; that is, not only $u_{i}$ and $B_{i}$ but also $u_{i+1}$ and $B_{i}$ are connected. This proves that $u$ and $v$ have a contact.

By Lemma 10, we obtain the following theorem immediately, which shows that the connectivity of wireless network systems and the connectivity of covering approximation spaces are equivalent.

Theorem 11. Let $(U ; \mathscr{B})$ be a wireless network system, and let $(U ; \mathscr{C})$ be a covering approximation space induced by $(U ; \mathscr{B})$. Then the following are equivalent.

(1) $(U ; \mathscr{B})$ is connected.

(2) $(U ; \mathscr{C})$ is connected.

We give a simple example to illustrate an application of Theorem 11.

Example 12. Let $\mathscr{B}=\left\{B_{1}, B_{2}, B_{3}\right\}$ be the family of three base stations and let $U=\left\{u_{1}, u_{2}, u_{3}, u_{4}, u_{5}, u_{6}\right\}$ be the set of some users, where $u_{1}$ (resp., $u_{2}$ ) and $B_{1}$ are connected, $u_{2}$ (resp., $u_{3}, u_{4}$ ) and $B_{2}$ are connected, and $u_{3}$ (resp., $u_{5}$, $\left.u_{6}\right)$ and $B_{3}$ are connected. Then $(U ; \mathscr{B})$ is a wireless network system. Put $K_{1}=\left\{u_{1}, u_{2}\right\}, K_{2}=\left\{u_{2}, u_{3}, u_{4}\right\}, K_{3}=$ $\left\{u_{3}, u_{5}, u_{6}\right\}$, and $\mathscr{C}=\left\{K_{1}, K_{2}, K_{3}\right\}$. It is clear that $(U ; \mathscr{C})$ is a covering approximation space induced by $(U ; \mathscr{B})$. For each pair $u_{i}, u_{j} \in U$, it is not difficult to check that $u_{i}$ and $u_{j}$ are connected in $(U ; \mathscr{C})$. So $(U ; \mathscr{C})$ is connected. By Theorem 11 , $(U ; \mathscr{B})$ is connected.

\section{The Connectivity of Covering Approximation Spaces}

As a classical result in topology, a topological space $(X, \mathscr{T})$ is connected if and only if $(X, \mathscr{T})$ has no nonempty clopen (i.e., both open and closed) proper subset. How can we characterize the connectivity of covering approximation spaces? This is an interesting question, which is still open. Note that there are no concepts for open subset and closed subset in covering approximation spaces. This shows that we need to find some subsets of covering approximation spaces to characterize the connectivity of covering approximation spaces. Similar to open subsets, closed subsets, and clopen subsets in topological spaces, there are three concepts generated by Pawlak approximation operators in Pawlak's models, which are definable subsets, inner definable subsets, and outer definable subsets (see, e.g., [26]). This leads us to generalize these concepts by covering approximation operators from Pawlak's models to covering approximation spaces and to characterize the connectivity of covering approximation spaces by these subsets. It is known that there are many covering approximation operators on covering approximation spaces (see, e.g., [19]). However, our discussion will be around the following covering upper approximation operator and covering lower approximation operator, which are important and effective in study for covering approximation spaces and were used frequently in discussions for covering approximation spaces (see, e.g., $[1,9,15,22]$ ).

Definition 13. Let $(U ; \mathscr{C})$ be a covering approximation space. For each $X \subseteq U$, put

$$
\begin{aligned}
& \underline{C}(X)=\{x \in U: \forall K \in \mathscr{C}(x \in K \Longrightarrow K \subseteq X)\} ; \\
& \bar{C}(X)=\bigcup\{K: K \in \mathscr{C} \bigwedge K \bigcap X \neq \emptyset\} .
\end{aligned}
$$

(1) $\underline{C}: 2^{U} \rightarrow 2^{U}$ is called covering lower approximation operator, and $\underline{C}(X)$ is called a covering lower approximation of $X$.

(2) $\bar{C}: 2^{U} \rightarrow 2^{U}$ is called covering upper approximation operator, and $\bar{C}(X)$ is called a covering upper approximation of $X$.

The following lemma comes from [9].

Lemma 14. Let $(U ; \mathscr{C})$ be a covering approximation space and $X \subseteq U$. Then $\underline{C}(X) \subseteq X \subseteq \bar{C}(X)$.

Definition 15. Let $(U ; \mathscr{C})$ be a covering approximation space and $X \subseteq U$.

(1) $X$ is called a definable subset of $(U ; \mathscr{C})$ if $\bar{C}(X)=$ $\underline{C}(X)$.

(2) $X$ is called an inner definable subset of $(U ; \mathscr{C})$ if $\underline{C}(X)=X$.

(3) $X$ is called an outer definable subset of $(U ; \mathscr{C})$ if $\bar{C}(X)=X$. 
Let $X$ be a subset of a covering approximation space $(U ; \mathscr{C})$. By Lemma $14, X$ is a definable subset of $(U ; \mathscr{C})$ if and only if $X$ is both an inner definable and an outer definable subset of $(U ; \mathscr{C})$. In fact, we have the better result.

Proposition 16. Let $(U ; \mathscr{C})$ be a covering approximation space and $X \subseteq U$. Then the following are equivalent.

(1) $X$ is a definable subset of $(U ; \mathscr{C})$.

(2) $X$ is an inner definable subset of $(U ; \mathscr{C})$.

(3) $X$ is an outer definable subset of $(U ; \mathscr{C})$.

Proof. $(1) \Rightarrow(2)$ : it holds from Lemma 14 .

$(2) \Rightarrow(3)$ : let $X$ be an inner definable subset of $(U ; \mathscr{C})$, that is, $\underline{C}(X)=X$. It suffices to prove that $\bar{C}(X)=X$. By Lemma 14 , we only need to prove that $\bar{C}(X) \subseteq X$. Let $u \in$ $\bar{C}(X)$. Then there is $K_{u} \in \mathscr{C}$ such that $u \in K_{u}$ and $K_{u} \cap X \neq \emptyset$. Pick $v \in K_{u} \bigcap X$; then $v \in X=\underline{C}(X)=\{w \in U: \forall K \in$ $\mathscr{C}(w \in K \Rightarrow K \subseteq X)\}$. Since $v \in K_{u}, K_{u} \subseteq X$, and, hence, $u \in K_{u} \subseteq X$. This proves that $\bar{C}(X) \subseteq X$.

$(3) \Rightarrow(1)$ : let $X$ be an outer definable subset of $(U ; \mathscr{C})$, that is, $\bar{C}(X)=X$. It suffices to prove that $C(X)=X$. By Lemma 14, we only need to prove that $X \subseteq \underline{C}(X)$. Let $u \in X$. For each $K \in \mathscr{C}$, if $u \in K$, then $u \in K \bigcap \bar{X} \neq \emptyset$, and hence $K \subseteq \bar{C}(X)=X$. It follows that $u \in \underline{C}(X)$. This proves that $X \subseteq \underline{C}(X)$.

Lemma 17. Let $(U ; \mathscr{C})$ be a covering approximation space and let $u \in U$. Put $X=\{s \in U: u$ is chain connected to $s\}$. If $X=U$, then $(U ; \mathscr{C})$ is connected.

Proof. Let $X=U$. Whenever $v, w \in U=X$, then $u$ is chain connected to $v$ and $u$ is chain connected to $w$. By Remark 8 , $v$ is chain connected to $w$. So $(U ; \mathscr{C})$ is connected.

Now we give the main theorem, which characterizes the connectivity of covering approximation spaces by their definable subsets.

Theorem 18. Let $(U ; \mathscr{C})$ be a covering approximation space. Then the following are equivalent.

(1) $(U ; \mathscr{C})$ is connected.

(2) $(U ; \mathscr{C})$ has no nonempty definable proper subset.

Proof. $(1) \Rightarrow(2)$. Suppose that $(U ; \mathscr{C})$ is connected. Let $X$ be a nonempty definable subset of $(U ; \mathscr{C})$. By Lemma $14, \bar{C}(X)=$ $\underline{C}(X)=X \neq \emptyset$. We only need to prove that $X$ is not a proper subset of $U$. Let $u \in U$. Pick $v \in X$; then $v$ is chain connected to $u$; that is, there are $K_{1}, K_{2}, \ldots, K_{n} \in \mathscr{C}$ such that $v \in K_{1}$, $u \in K_{n}$, and $K_{i} \cap K_{i+1} \neq \emptyset$ for each $i=1,2, \ldots, n-1$. Since $v \in K_{1} \bigcap X \neq \emptyset, K_{1} \subseteq \bar{C}(X)=X$. Furthermore, $K_{2} \bigcap X$ ว $K_{2} \cap K_{1} \neq \emptyset$, so $K_{2} \subseteq \bar{C}(X)=X$. In the same way, we can obtain that $K_{n} \subseteq \bar{C}(X)=X$. Thus, $u \in K_{n} \subseteq X$. This proves that $U=X$. So $X$ is not a proper subset of $U$.

$(2) \Rightarrow(1)$. Suppose that $(U ; \mathscr{C})$ has no nonempty definable proper subset. Let $u \in U$. Put $X=\{s \in U$ : $u$ is chain connected to $s\}$. Then $u \in X \neq \emptyset$ by Remark $8(1)$. Whenever $v \in \bar{C}(X)$, there is $K \in \mathscr{C}$ such that $K \bigcap X \neq \emptyset$ and $v \in K$. Pick $w \in K \bigcap X$. Then $u$ is chain connected to $w$, and $w$ is chain connected to $v$. So $u$ is chain connected to $v$ by Remark $8(3)$. It follows that $v \in X$. This proves that $\bar{C}(X) \subseteq X$. On the other hand, $X \subseteq \bar{C}(X)$ from Lemma 14, and hence $\bar{C}(X)=X$. Thus, $X$ is an outer definable subset of $(U ; \mathscr{C})$. By Proposition $16, X$ is a definable subset of $(U ; \mathscr{C})$. It follows that $X=U$. By Lemma $17,(U ; \mathscr{C})$ is connected.

We give a simple example to illustrate an application of Theorem 18.

Example 19. Let $U=\left\{u_{1}, u_{2}, u_{3}, u_{4}, u_{5}, u_{6}\right\}$ be the universe of discourse. Put $K_{1}=\left\{u_{1}, u_{2}, u_{5}\right\}, K_{2}=\left\{u_{3}, u_{4}\right\}, K_{3}=$ $\left\{u_{3}, u_{6}\right\}$, and $\mathscr{C}=\left\{K_{1}, K_{2}, K_{3}\right\}$. Then $(U ; \mathscr{C})$ is a covering approximation space. Put $X=\left\{u_{3}, u_{4}, u_{6}\right\}$. Then $\bar{C}(X)=$ $\bigcup\{K: K \in \mathscr{C} \wedge K \bigcap X \neq \emptyset\}=\left\{u_{3}, u_{4}\right\} \bigcup\left\{u_{3}, u_{6}\right\}=$ $\left\{u_{3}, u_{4}, u_{6}\right\}=X$. So $X$ is a nonempty outer definable proper subset of $(U ; \mathscr{C})$. By Proposition $16, X$ is a nonempty definable proper subset of $(U ; \mathscr{C})$. It follows that $(U ; \mathscr{B})$ is not connected by Theorem 18 .

\section{An Application}

In this section, we give an application to show that our approach does work. This work is to assess the connectivity of a teacher-student interactive platform.

(1) The Teacher-Student Interactive Platform $(U ; \mathscr{B})$. The teacher-student interactive platform $(U ; \mathscr{B})$ is established in the School of Mathematical Sciences of Soochow University, which creates a new environment for the current students in the School of Mathematical Sciences of Soochow University and would promote the interaction among these students.

(1.1) $U$ is the set of twelve information points, which is denoted by $U=\left\{u_{i}: i=1,2, \ldots, 12\right\}$.

(1.2) $\mathscr{B}$ is the family of six information stations, which is denoted by $\mathscr{B}=\left\{B_{a}, B_{b}, B_{c}, B_{d}, B_{e}, B_{f}\right\}$.

(1.3) We call that an information point $u$ in $U$ and an information station $B$ in $\mathscr{B}$ are connected if $u$ and $B$ can receive and send information from and to each other. By restrictions of campus network for Soochow University, we can not make $u$ and $B$ connected for each information point $u$ in $U$ and for each information station $B$ in $\mathscr{B}$. However, the following are satisfied.

(1.3.1) $u_{1}$ and $B_{\alpha}$ are connected for $\alpha \in\{a, c, d\}$.

(1.3.2) $u_{2}$ and $B_{\alpha}$ are connected for $\alpha \in\{b, c, f\}$.

(1.3.3) $u_{3}$ and $B_{\alpha}$ are connected for $\alpha \in\{b, d, e\}$.

(1.3.4) $u_{4}$ and $B_{\alpha}$ are connected for $\alpha \in\{d, e, f\}$.

(1.3.5) $u_{5}$ and $B_{\alpha}$ are connected for $\alpha \in\{a, b, f\}$.

(1.3.6) $u_{6}$ and $B_{\alpha}$ are connected for $\alpha \in\{a, e, f\}$.

(1.3.7) $u_{7}$ and $B_{\alpha}$ are connected for $\alpha \in\{a, c, e\}$.

(1.3.8) $u_{8}$ and $B_{\alpha}$ are connected for $\alpha \in\{a, b, e\}$.

(1.3.9) $u_{9}$ and $B_{\alpha}$ are connected for $\alpha \in\{c, d, e\}$. 
TABLE 1: The teacher-student interactive platform $(U ; \mathscr{B})$.

\begin{tabular}{ccccccccccccc}
\hline & $u_{1}$ & $u_{2}$ & $u_{3}$ & $u_{4}$ & $u_{5}$ & $u_{6}$ & $u_{7}$ & $u_{8}$ & $u_{9}$ & $u_{10}$ & $u_{11}$ & $u_{12}$ \\
\hline$B_{a}$ & 1 & 0 & 0 & 0 & 1 & 1 & 1 & 1 & 0 & 0 & 1 \\
$B_{b}$ & 0 & 1 & 1 & 0 & 1 & 0 & 0 & 1 & 0 & 1 & 0 \\
$B_{c}$ & 1 & 1 & 0 & 0 & 0 & 0 & 1 & 0 & 1 & 0 & 1 \\
$B_{d}$ & 1 & 0 & 1 & 1 & 0 & 0 & 0 & 0 & 1 & 1 & 0 \\
$B_{e}$ & 0 & 0 & 1 & 1 & 0 & 1 & 1 & 1 & 1 & 0 & 0 \\
$B_{f}$ & 0 & 1 & 0 & 1 & 1 & 1 & 0 & 0 & 0 & 1 & 0 \\
\hline
\end{tabular}

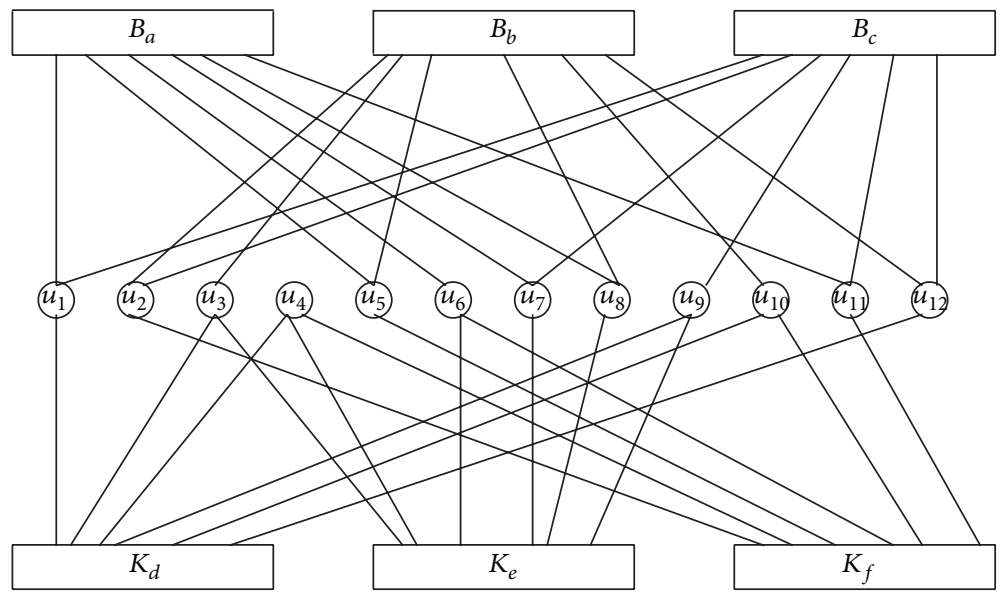

Figure 1

(1.3.10) $u_{10}$ and $B_{\alpha}$ are connected for $\alpha \in\{b, d, f\}$.

(1.3.11) $u_{11}$ and $B_{\alpha}$ are connected for $\alpha \in\{a, c, f\}$.

(1.3.12) $u_{12}$ and $B_{\alpha}$ are connected for $\alpha \in\{b, c, d\}$.

The above connectivity can also be described as shown in Figure 1.

(1.4) By Definition 1, it is not difficult to check that the teacher-student interactive platform $(U ; \mathscr{B})$ forms a wireless network system, which can be described as in Table 1. Here, $U=\left\{u_{i}: i=1,2, \ldots, 12\right\}$, $\mathscr{B}=\left\{B_{a}, B_{b}, B_{c}, B_{d}, B_{e}, B_{f}\right\}$, and the number, which lies in the cross of the row labeled by $B(B=$ $\left.B_{a}, B_{b}, B_{c}, B_{d}, B_{e}, B_{f}\right)$ and the column labeled by $x$ $\left(x=u_{1}, u_{2}, u_{3}, u_{4}, u_{5}, u_{6}, u_{7}, u_{8}, u_{9}, u_{10}, u_{11}, u_{12}\right)$, is 1 or 0 by $x$ and $B$ are connected or $x$ and $B$ are not connected.

(1.5) If the teacher-student interactive platform $(U ; \mathscr{B})$ is connected, then students can communicate easily with each other by using $(U ; \mathscr{B})$.

(2) The Covering Approximation Space $(U ; \mathscr{C})$ Induced by $(U ; \mathscr{B})$

(2.1) For each $\alpha \in\{a, b, c, d, e, f\}$, let $K_{\alpha}$ be a set of some information points in $U$ such that $u$ is an information point in $K_{\alpha}$ if and only if $u$ and $B_{\alpha}$ are connected:

(2.1.1) $K_{a}=\left\{u_{1}, u_{5}, u_{6}, u_{7}, u_{8}, u_{11}\right\}$,

(2.1.2) $K_{b}=\left\{u_{2}, u_{3}, u_{5}, u_{8}, u_{10}, u_{12}\right\}$,
(2.1.3) $K_{c}=\left\{u_{1}, u_{2}, u_{7}, u_{9}, u_{11}, u_{12}\right\}$,

(2.1.4) $K_{d}=\left\{u_{1}, u_{3}, u_{4}, u_{9}, u_{10}, u_{12}\right\}$,

(2.1.5) $K_{e}=\left\{u_{3}, u_{4}, u_{6}, u_{7}, u_{8}, u_{9}\right\}$,

(2.1.6) $K_{f}=\left\{u_{2}, u_{4}, u_{5}, u_{6}, u_{10}, u_{11}\right\}$.

(2.2) Put $\mathscr{C}=\left\{K_{a}, K_{b}, K_{c}, K_{d}, K_{e}, K_{f}\right\}$.

(2.3) It is clear that $\mathscr{C}$ is a cover of $U$. By Proposition 5 and Definition $6,(U ; \mathscr{C})$ is a covering approximation space induced by $(U ; \mathscr{B})$.

(3) The Connectivity of $(U ; \mathscr{C})$. By a simple algorithm, it can be obtained that if $X$ is a nonempty outer definable subset of $(U ; \mathscr{C})$, then $X=U$. In fact, let $X$ be an outer definable subset of $(U ; \mathscr{C})$ and $X \neq \emptyset$. Then there is $u_{i} \in X$ for some $i \in$ $\{1,2, \ldots, 12\}$. If $u_{1} \in X$, then $K_{\alpha} \cap X \neq \emptyset$ for $\alpha=a, c, d$. Thus, $=\bar{C}(X)=\bigcup\{K: K \in \mathscr{C} \wedge K \bigcap X \neq \emptyset\} \supseteq K_{a} \bigcup K_{c} \bigcup K_{d}=$ $\left\{u_{1}, u_{2}, u_{3}, u_{4}, u_{5}, u_{6}, u_{7}, u_{8}, u_{9}, u_{10}, u_{11}, u_{12}\right\}=U$. It follows that $X=U$. By the same method, we can obtain that if $u_{i} \in X$ for any $i \in\{2,3, \ldots, 12\}$, then $X=U$. This shows that $(U ; \mathscr{C})$ has no nonempty outer definable proper subset. By Proposition $16,(U ; \mathscr{C})$ has no nonempty definable proper subset. It follows that $(U ; \mathscr{C})$ is connected from Theorem 18 .

(4) The Connectivity of $(U ; \mathscr{B})$. By Theorem $11,(U ; \mathscr{B})$ is connected.

By (1.5), the students can communicate easily with each other by using the teacher-student interactive platform $(U ; \mathscr{B})$. 
Remark 20. By teacher-student interactive platforms, we give a further application of rough set theory in pedagogy, which makes it possible to research education by logical methods and mathematical methods.

\section{Conclusions}

In this paper, we introduce wireless network systems and take covering approximation spaces as mathematical models of wireless network systems. We prove that a wireless network system is connected if and only if the relevant covering approximation space is connected. With the help of covering approximation operators $\bar{C}$ and $\underline{C}$, we characterize the connectivity of covering approximation spaces by their definable subsets. Then, it is obtained that a wireless network system is connected if and only if the relevant covering approximation space has no nonempty definable proper subset. As a concrete application of covering approximation spaces in wireless network systems, we discuss the connectivity of teacherstudent interactive platforms, which further demonstrates the usefulness of rough set theory in pedagogy and makes it possible to research education by logical methods and mathematical methods.

\section{Conflict of Interests}

The author declares that there is no conflict of interests regarding the publication of this paper.

\section{Acknowledgments}

The author wishes to thank the reviewers for reviewing this paper and offering their valuable comments. This work is supported by the National Natural Science Foundation of China (no. 11301367), Doctoral Fund of Ministry of Education of China (no. 20123201120001), China Postdoctoral Science Foundation (no. 2013M541710), and Jiangsu Province Postdoctoral Science Foundation (no. 1302156C).

\section{References}

[1] W. Zhu and F.-Y. Wang, "On three types of covering-based rough sets," IEEE Transactions on Knowledge and Data Engineering, vol. 19, no. 8, pp. 1131-1143, 2007.

[2] Z. Pawlak, "Rough sets," International Journal of Computer and Information Sciences, vol. 11, no. 5, pp. 341-356, 1982.

[3] Z. Cai, X. Guan, P. Shao, Q. Peng, and G. Sun, "A rough set theory based method for anomaly intrusion detection in computer network systems," Expert Systems, vol. 20, no. 5, pp. 251-259, 2003.

[4] D. Ciucci and T. Flaminio, "Generalized rough approximations in $L \Pi(1 / 2)$," International Journal of Approximate Reasoning, vol. 48, no. 2, pp. 544-558, 2008.

[5] K. Kaneiwa, "A rough set approach to multiple dataset analysis," Applied Soft Computing, vol. 11, no. 2, pp. 2538-2547, 2011.

[6] Y. Leung, W.-Z. Wu, and W.-X. Zhang, "Knowledge acquisition in incomplete information systems: a rough set approach," European Journal of Operational Research, vol. 168, no. 1, pp. 164-180, 2005.
[7] Z. Pawlak, Rough Sets: Theoretical Aspects of Reasoning about Data, Kluwer Academic Publishers, Boston, Mass, USA, 1991.

[8] Z. Pawlak, “Rough classification," International Journal of Human Computer Studies, vol. 51, no. 2, pp. 369-383, 1999.

[9] J. A. Pomykała, "Approximation operations in approximation space," Bulletin of the Polish Academy of Sciences. Mathematics, vol. 35, no. 9-10, pp. 653-662, 1987.

[10] A. Skowron and J. F. Peters, "Rough-Granular computing," in Hand-Book of Granular Computing, W. Pedrycz, A. Skowron, and V. Kreinovich, Eds., pp. 285-328, John Wiley \& Sons, 2008.

[11] R. Slowinski and D. Vanderpooten, "A generalized definition of rough approximations based on similarity," IEEE Transactions on Knowledge and Data Engineering, vol. 12, no. 2, pp. 331-336, 2000.

[12] Y. Y. Yao, "Two views of the theory of rough sets in finite universes," International Journal of Approximate Reasoning, vol. 15, no. 4, pp. 291-317, 1996.

[13] Z. Bonikowski, E. Bryniarski, and U. Wybraniec-Skardowska, "Extensions and intentions in the rough set theory," Information Sciences, vol. 107, no. 1-4, pp. 149-167, 1998.

[14] X. Ge, "An application of covering approximation spaces on network security," Computers \& Mathematics with Applications, vol. 60, no. 5, pp. 1191-1199, 2010.

[15] X. Ge, X. Bai, and Z. Yun, "Topological characterizations of covering for special covering-based upper approximation operators," Information Sciences, vol. 204, pp. 70-81, 2012.

[16] X. Ge and Z. Li, "Definable subsets in covering approximation spaces," International Journal of Computational and Mathematical Sciences, vol. 5, no. 1, pp. 31-34, 2011.

[17] E. F. Lashin, A. M. Kozae, A. A. Abo Khadra, and T. Medhat, "Rough set theory for topological spaces," International Journal of Approximate Reasoning, vol. 40, no. 1-2, pp. 35-43, 2005.

[18] K. Qin, Y. Gao, and Z. Pei, “On covering rough sets," in Rough Sets and Knowledge Technology, vol. 4481 of Lecture Notes in Computer Science, pp. 34-41, Springer, Berlin, Germany, 2007.

[19] P. Samanta and M. K. Chakraborty, "Covering based approaches to rough sets and implication lattices," in Rough Sets, Fuzzy Sets, Data Mining and Granular Computing, vol. 5908 of Lecture Notes in Computer Science, pp. 127-134, Springer, Berlin, Germany, 2009.

[20] A. Skowron and J. Stepaniuk, "Tolerance approximation spaces," Fundamenta Informaticae, vol. 27, no. 2-3, pp. 245-253, 1996.

[21] Y. Y. Yao, "On generalizing rough set theory," in Rough Sets, Fuzzy Sets, Data Mining, and Granular Computing, vol. 2639 of Lecture Notes in Computer Science, pp. 44-51, Springer, Berlin, Germany, 2003.

[22] Z. Yun, X. Ge, and X. Bai, "Axiomatization and conditions for neighborhoods in a covering to form a partition," Information Sciences, vol. 181, no. 9, pp. 1735-1740, 2011.

[23] N. Zhong, Y. Yao, and M. Ohshima, "Peculiarity oriented multidatabase mining," IEEE Transactions on Knowledge and Data Engineering, vol. 15, no. 4, pp. 952-960, 2003.

[24] Á. Császár, “Generalized topology, generalized continuity," Acta Mathematica Hungarica, vol. 96, no. 4, pp. 351-357, 2002.

[25] R. Engelking, General Topology, vol. 6 of Sigma Series in Pure Mathematics, Heldermann, Berlin, Germany, 2nd edition, 1989.

[26] D. Pei, “On definable concepts of rough set models," Information Sciences, vol. 177, no. 19, pp. 4230-4239, 2007. 


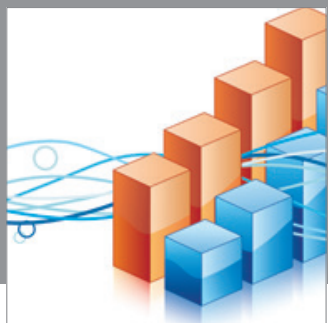

Advances in

Operations Research

mansans

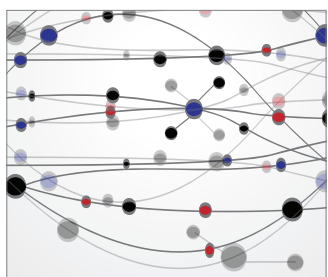

The Scientific World Journal
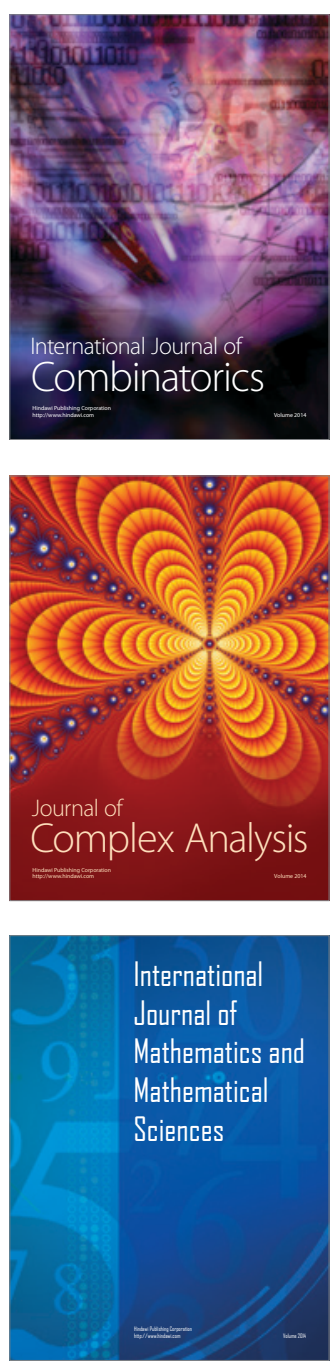
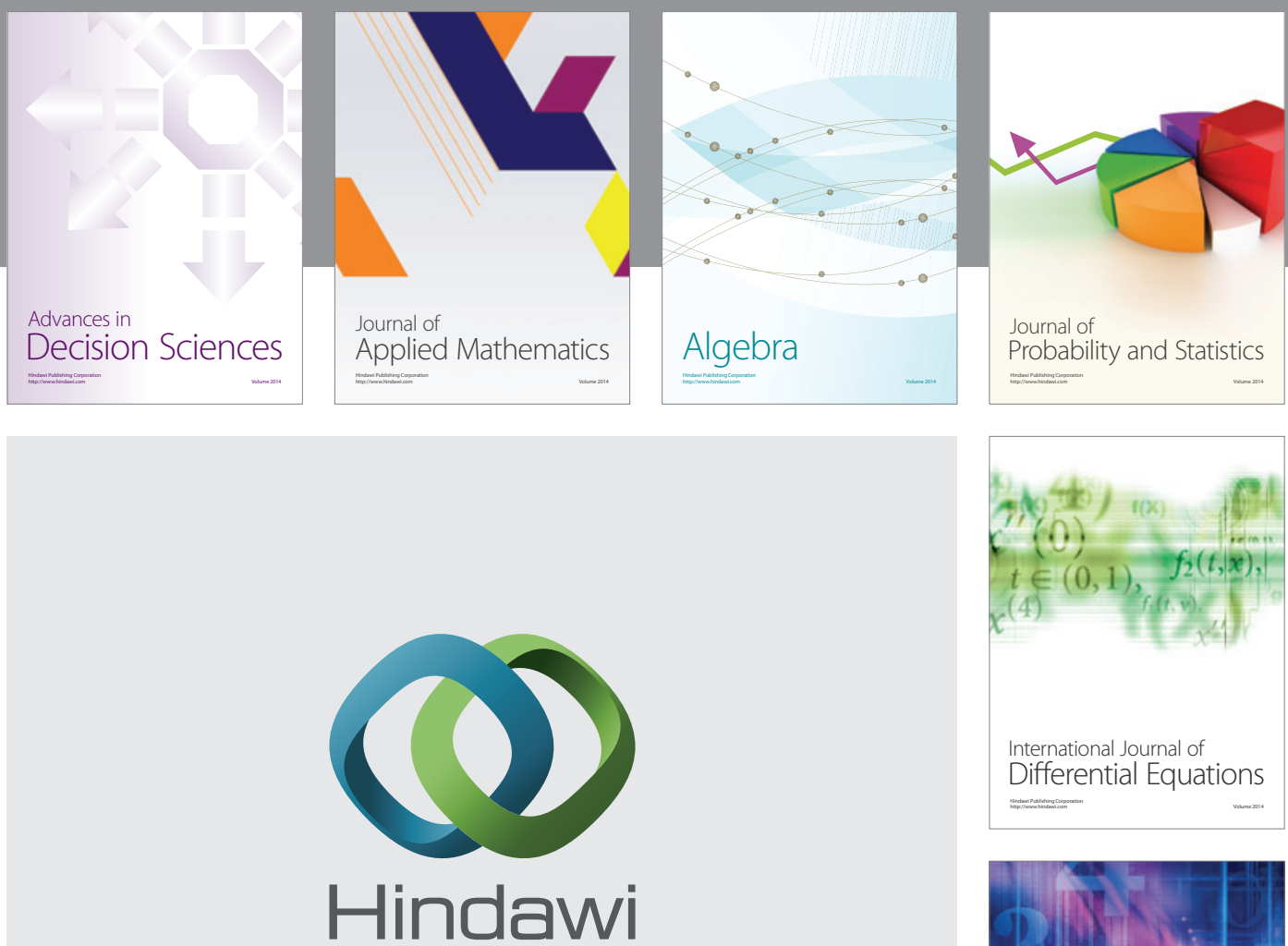

Submit your manuscripts at http://www.hindawi.com
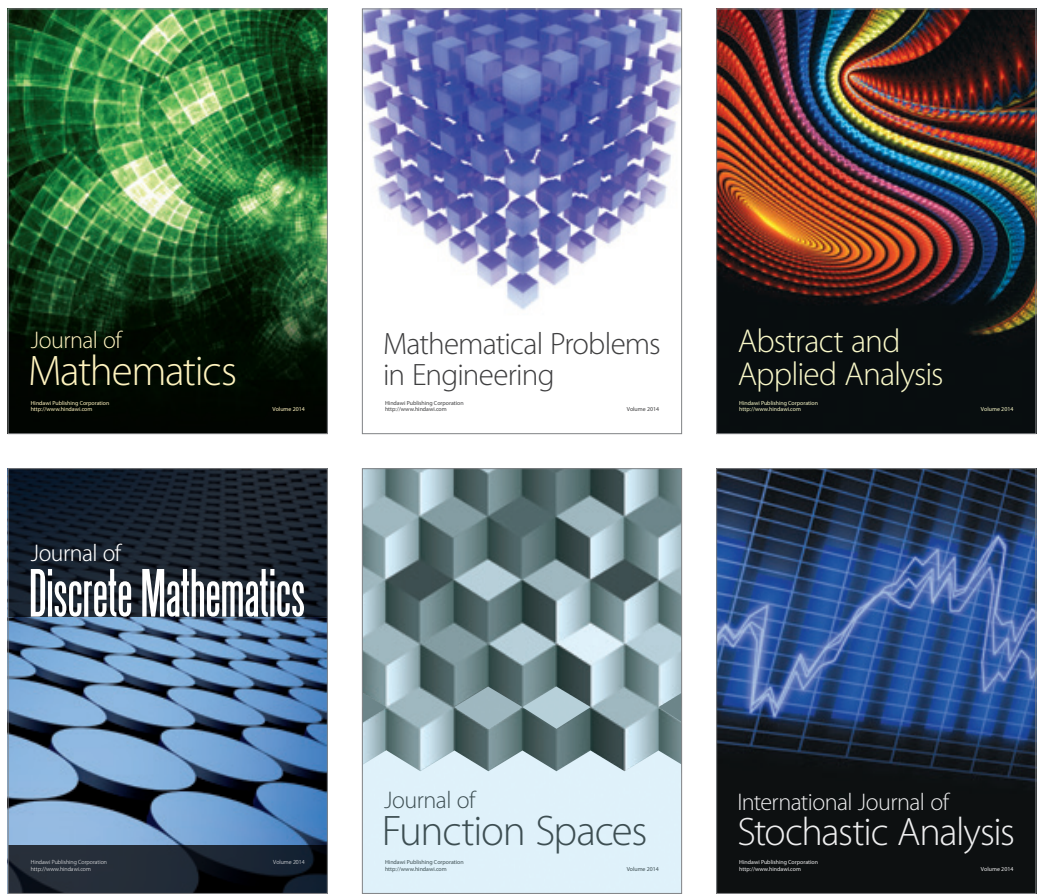

Journal of

Function Spaces

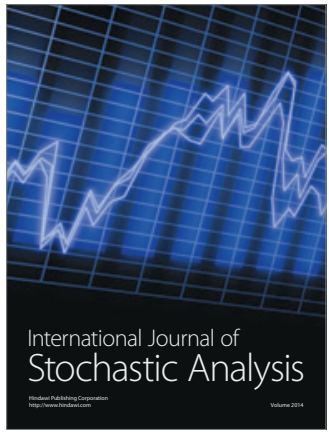

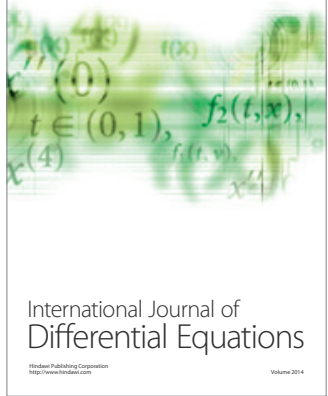
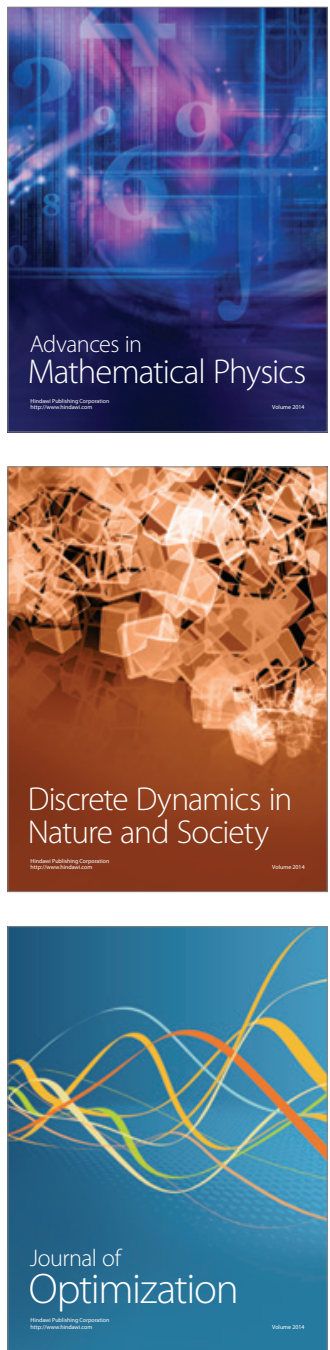